\title{
The Women's wellness after cancer program: a multisite, single-blinded, randomised controlled trial protocol
}

\author{
Debra Anderson ${ }^{1 *}$, Charrlotte Seib ${ }^{1,2}$, Dian Tjondronegoro ${ }^{2}$, Jane Turner ${ }^{3,4}$, Leanne Monterosso ${ }^{5,6}$, \\ Amanda McGuire ${ }^{2}$, Janine Porter-Steele ${ }^{1,2,7}$, Wei Song ${ }^{8}$, Patsy Yates ${ }^{2}$, Neil King ${ }^{2}$, Leonie Young ${ }^{7}$, Kate White ${ }^{9,10}$, \\ Kathryn Lee ${ }^{11}$, Sonj Hall ${ }^{12}$, Mei Krishnasamy ${ }^{13}$, Kathy Wells ${ }^{14}$, Sarah Balaam ${ }^{2}$ and Alexandra L. McCarthy ${ }^{15,2}$
}

\begin{abstract}
Background: Despite advances in cancer diagnosis and treatment have significantly improved survival rates, patients post-treatment-related health needs are often not adequately addressed by current health services. The aim of the Women's Wellness after Cancer Program (WWACP), which is a digitised multimodal lifestyle intervention, is to enhance health-related quality of life in women previously treated for blood, breast and gynaecological cancers.

Methods: A single-blinded, multi-centre randomized controlled trial recruited a total of 330 women within 24 months of completion of chemotherapy (primary or adjuvant) and/or radiotherapy. Women were randomly assigned to either usual care or intervention using computer-generated permuted-block randomisation. The intervention comprises an evidence-based interactive iBook and journal, web interface, and virtual health consultations by an experienced cancer nurse trained in the delivery of the WWACP. The 12 week intervention focuses on evidence-based health education and health promotion after a cancer diagnosis. Components are drawn from the American Cancer Research Institute and the World Cancer Research Fund Guidelines (2010), incorporating promotion of physical activity, good diet, smoking cessation, reduction of alcohol intake, plus strategies for sleep and stress management. The program is based on Bandura's social cognitive theoretical framework. The primary outcome is health-related quality of life, as measured by the Functional Assessment of Cancer Therapy-General (FACT-G). Secondary outcomes are menopausal symptoms as assessed by Greene Climacteric Scale; physical activity elicited with the Physical Activity Questionnaire Short Form (IPAQ-SF); sleep measured by the Pittsburgh Sleep Quality Index; habitual dietary intake monitored with the Food Frequency Questionnaire (FFQ); alcohol intake and tobacco use measured by the Australian Health Survey and anthropometric measures including height, weight and waist-to-hip ratio. All participants were assessed with these measures at baseline (at the start of the intervention), 12 weeks (at completion of the intervention), and 24 months (to determine the level of sustained behaviour change). Further, a simultaneous cost-effectiveness evaluation will consider if the WWACP provides value for money and will be reported separately.
\end{abstract}

Discussion: Women treated for blood, breast and gynaecological cancers demonstrate increasingly good survival rates. However, they experience residual health problems that are potentially modifiable through behavioural lifestyle interventions such as the WWACP.

Trial registration: The protocol for this study was registered with the Australian and New Zealand Clinical Trials Registry, Trial ID: ACTRN12614000800628, July 28, 2014.

Keywords: Cancer, Women, health-related quality of life, Menopausal symptoms, Modifiable lifestyle factors

\footnotetext{
* Correspondence: debra.anderson@griffith.edu.au

${ }^{1}$ Menzies Health Institute Queensland, Griffith University, Menzies Health

Institute Queensland, Parklands Drive, Southport, Queensland 4215, Australia

Full list of author information is available at the end of the article
}

(c) The Author(s). 2017 Open Access This article is distributed under the terms of the Creative Commons Attribution 4.0 International License (http://creativecommons.org/licenses/by/4.0/), which permits unrestricted use, distribution, and reproduction in any medium, provided you give appropriate credit to the original author(s) and the source, provide a link to the Creative Commons license, and indicate if changes were made. The Creative Commons Public Domain Dedication waiver (http://creativecommons.org/publicdomain/zero/1.0/) applies to the data made available in this article, unless otherwise stated. 


\section{Background}

While the overall incidence of female cancers is increasing, advances in diagnosis and treatment have significantly improved survival rates. In some cases, this has transformed cancer from an often fatal condition to a chronic and often curable disease (for example, early breast malignancies) [1]. Although survival rates are improving, survival can involve a number of treatmentrelated health problems including ovarian failure [2], weight gain [3], cognitive alterations [4], and fatigue [5]. For women after cancer, these physical and psychological sequelae can be severe and frequently adversely affect their quality of life [2].

Recent studies indicate that many survivorship-related health problems can be modified through lifestyle practices such as good diet, greater physical activity, and participation in screening programs, though cancer survivors do not necessarily adopt these behaviours [6, 7]. According to two large American studies, few people treated for cancer met recommendations for smoking cessation, alcohol minimisation, physical activity, or fruit and vegetable intake $[6,8]$. Conversely, our pilot work indicates that many cancer survivors are highly motivated to protect their health and are more likely to take up beneficial lifestyle and risk reduction strategies if they are targeted towards the end of or soon after completion of treatment $[9,10]$.

Australian health services and cancer support organisations do not often provide structured health promotion programs to support cancer survivors to minimise lifestyle-related health risks once they have ceased active treatment, despite the public and personal health benefits that these would provide. While a range of survivorship programs in recent years have recognised the chronic nature of cancer, most are narrowly targeted on specific symptoms and not based on sound chronic disease self-management principles and the management of risk through lifestyle [11]. The need for support is particularly acute among rural and outer metropolitan survivors, who have restricted access to face-to-face services due to cost, time, geographical, and other barriers. Novel approaches to promote beneficial lifestyle practices in women after cancer, such as the WWACP, have the potential to improve quality of life and reduce chronic disease risk.

The aim of this randomised controlled trial (RCT) is to determine the efficacy and cost effectiveness of a multimodal, digitised lifestyle intervention - The Women's Wellness after Cancer Program or WWACP. The WWACP aims to enhance health-related quality of life in women previously treated for breast, gynaecological, or blood cancers. The hypotheses are that, compared to usual care, women undertaking the WWACP intervention will:
1. Demonstrate higher scores of HRQoL irrespective of their place of residence.

2. Be more likely to have a body mass index (BMI) within recommended healthy weight range (e.g. BMI 20-25, waist circumference $<80 \mathrm{~cm}$ )

3. Show greater long term adherence to diet, exercise, sleep, alcohol, and smoking recommendations.

4. Evaluate if the WWACP provides better value for money than the current usual care approaches (reported elsewhere).

\section{Methods}

This multi-centre, single-blinded, randomized controlled 12-week trial involved five hospitals (public and private) and consumer groups serving women from metropolitan and non-metropolitan areas throughout Australia. The study was funded by a National Health and Medical Research Council (NHMRC) Partnership Grant (APP1056856). It received ethical approval from the relevant Institutional Review Boards of participating sites before commencing the trial. Details of the trial are further illustrated in Fig. 1.

\section{Sampling and recruitment}

The study population included women aged 18 and treated for breast, blood or gynaecological cancer within the previous 24 months. These cancers were chosen as they comprise approximately $46 \%$ of all female cancers in Australia in 2007 [1] and their treatment is associated with numerous ongoing health complaints. These problems include menopausal symptoms, osteopenia and osteoporosis, obesity, diabetes, cognitive alterations, peripheral neuropathy and cardiac health consequences. Good evidence indicates that many of these comorbidities can be prevented or mitigated by consistent engagement in health promoting activities and weight reduction $[10,12-$ 15], which are the foci of this intervention.

Participants were recruited by clinicians from five hospital sites: Princess Alexandra Hospital, Royal Brisbane and Women's Hospital, St John of God Murdoch Hospital, Peter MacCallum Cancer Institute, and the Choices Cancer Support Program; and through newsletters, emails and websites of three consumer groups (the National Breast Cancer Foundations' Register4, CanSpeak Queensland, and the Breast Cancer Network of Australia).

Inclusion criteria included completion of chemotherapy (primary or adjuvant) and/or radiotherapy within the previous 24 months for breast, blood, or gynaecological cancer; English proficiency; and access to an Apple computer and or iPad (due to interactive book's comparability). Participants were excluded if they had metastatic or advanced cancer, inoperable or active loco-regional disease, were on maintenance chemotherapy for blood cancers. 


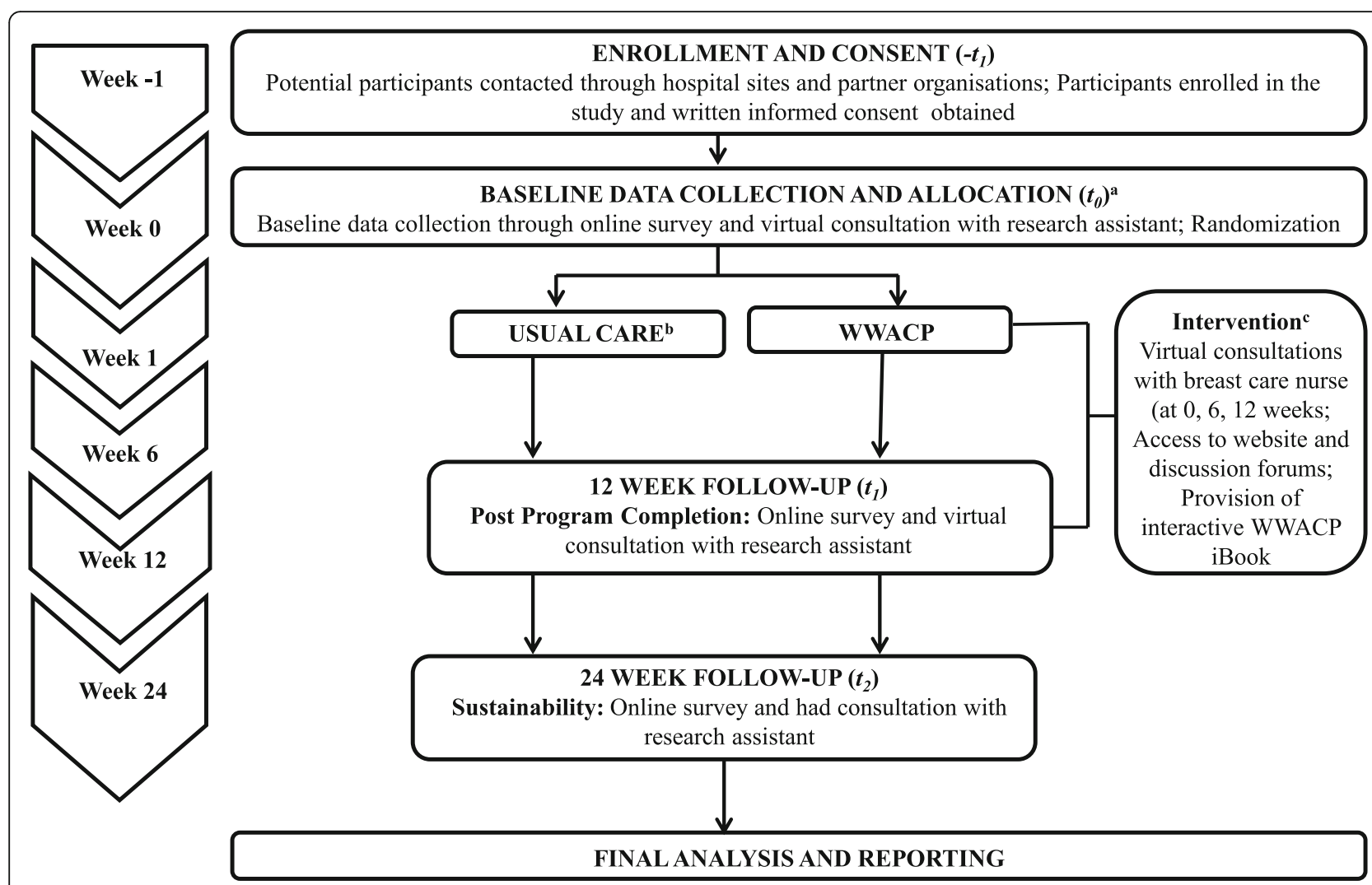

Fig. 1 Flowchart for the WWACP study. Notes: ${ }^{\text {a }}$ All participants completed a structured online questionnaire and virtual consultation with a research assistant at baseline (t0), 12 weeks $(t 1)$ and 24 weeks $(t 2){ }^{\mathbf{b}}$ The control group received general information only; ${ }^{\mathbf{c}}$ The intervention group received intervention materials and three virtual consultations with a breast cancer nurse at baseline (t0), 6 weeks (mid-intervention), and 12 weeks ( $t 1$ )

\section{Randomisation}

The RCT comprises two treatments (intervention vs usual care) and three time points for data collection; baseline (t0), 12 weeks (t1) and 24 weeks (t2). The procedure for random allocation occurred after baseline data collection and was performed by a member of the research team independent of recruitment or data collection. Permuted-block randomisation was used, with the seed for the random number generator determined by entering the number of treatments, and the numbers of patients per specific block (i.e., with two treatment groups and block sizes ranging from 2-8). The exact sequence was generated using algorithms [16, 17] available at http://www.randomization.com.

\section{Intervention}

This multi-modal intervention was designed for delivery via an e-health enabled platform. It comprises virtuallydelivered health professional consultations, an interactive web interface (including podcasts), an interactive electronic book $(i B o o k)$ which provides detailed intervention instructions and supports participants to log relevant health and lifestyle information into a journal.
The intervention targets health education and health promotion incorporating Australian and international recommendations for physical activity, diet, smoking cessation and minimising alcohol intake; as well as strategies to manage sleep and stress, menopausal symptoms, and sexual problems over a 12 week period (see Tables 1 and 2 for further detail). The timing and application of these strategies were tailored to meet patient's individual goals and functional capacities.

The iBook is structured around four chronological steps: changing lifestyle; establishing healthy habits; maintaining health for illness prevention; and becoming independent. More specifically, Step 1 provides essential information about a number of topics that form the basis of the 12-week program. Participants receive practical advice about how to incorporate this information into their lifestyle. Step 2 encourages participants to apply their new lifestyle habits to the areas of healthy weight, strong bones and hormonal change. Step 3 presents information about maintaining health for illness prevention. Step 3 emphasises a healthy heart, diabetes prevention, and cancer screening so that participants can manage their treatment-related risks for chronic disease in a proactive way. Finally, Step 4 focusses on 
Table 1 WWACP Intervention Content and Delivery Strategies

\begin{tabular}{lll}
\hline Week & Delivery Strategies & Content \\
\hline 1 & $\begin{array}{l}\text { Virtual consultation } \\
\text { delivered by specialist } \\
\text { cancer nurse }\end{array}$ & Physical activity, and healthy \\
& eating messages; goal setting; \\
& education; motivational \\
& interviewing; development of \\
& tailored health education and \\
& individualised plan and goals. \\
& Observational weight, and \\
& self-measured height, waist/hip \\
& circumference measures
\end{tabular}

2 Phone coaching, Journal, Review plan and goals; develop Book, health education a personal action plan; identify material/website barriers; self-monitoring

3 Phone coaching, Journal, Book, and website

Relapse prevention; coaching, feedback, motivational interviewing and self-monitoring.

4,12 Journal/website/SMS/e-mail Mobile phone text message every week based on program messages; news update every four weeks; motivational messages sent as women reach set goals,

6,12 Virtual RN consultation

1,12, 24 Data collection by RA

Review of plan and goals; coaching; relapse prevention; motivational interviewing; biophysical measurements. Observational weight and self-measured height, waist/hip circumference measures

Observational, weight, waist/hip circumference measures and on-line questionnaire

developing independence and sustaining healthy lifestyle changes after the 12-week program.

The interactive website provided healthy living support and home monitoring of measurable health indicators, which are downloaded to the study team. The website incorporates access to the downloadable $i B o o k$; educational podcasts and factsheets; a weekly exercise planner and schedule; and a community message board.

Virtual consultations were provided by experienced cancer nurses trained in the delivery of the WWACP, via skype or FaceTime at weeks 0, 6, and 12 (see Table 3 for further detail). These consultations included discussion and health education about physical activity, healthy eating, stress, sleep menopause and sexuality after cancer and strategies to promote intervention adherence. The women had $\mathrm{v}$ physical and psychological capabilities, the nurses therefore worked with them to set realistic goals within the woman's capacity.

\section{Standard care}

Participants allocated to the standard care group received general information from their usual health professionals during clinic visits about the management of all symptoms, including general advice about exercise, diet, tobacco and alcohol abstinence, plus information

Table 2 The Women's Wellness after Cancer Program targeted behaviours [40]

\begin{tabular}{ll}
\hline Targeted Behaviours & Rationale/Evidence \\
\hline Body Fatness: Be as lean as & Maintenance of a healthy weight \\
possible within the normal & may be one of the most important \\
weight range, avoid weight & ways to protect against cancer \\
gain and increases in waist & recurrence and other common \\
circumference & chronic diseases, including \\
& hypertension, stroke, type 2 diabetes \\
& and coronary heart disease [40]
\end{tabular}

Physical Activity: Be moderately physically active, equivalent to brisk walking, for at least $30 \mathrm{~min}$ per day; As fitness improves, aim for 60 min or more of moderate, or for 30 min or more of vigorous, physical activity every day

Diet: Eat mostly foods of plant origin; limit consumption of energy-dense foods; avoid sugary drinks, limit intake of red meat and avoid processed meat

Physical activity of longer duration or greater intensity is more beneficial; All forms of physical activity protect against some cancers, as well as against weight gain, overweight, and obesity [40]

An integrative approach to the evidence shows that most diets that are protective against cancer are mainly made up from foods of plant origin; Consumption of energy-dense foods and sugary drinks contributes to obesity; An integrated approach to the evidence also shows that many foods of animal origin are nourishing and healthy if consumed in modest amounts.

Alcohol: If alcoholic drinks are consumed limit consumption to no more

The evidence on cancer justifies a recommendation not to drink alcoholic drinks. Other evidence shows that modest amounts of alcoholic drinks are likely to reduce the risk of coronary heart disease [40]

Table 3 Consultation nurse delivery strategies and content

\begin{tabular}{|c|c|c|}
\hline Week & Delivery strategies & Content \\
\hline 1 & $\begin{array}{l}\text { Virtual consultation delivered } \\
\text { by specialist cancer nurse: } \\
\text { Phone coaching, iBook, } \\
\text { health education material, } \\
\text { website and email }\end{array}$ & $\begin{array}{l}\text { Introduction to website and } \\
\text { IBook. Physical activity, and } \\
\text { healthy eating messages; } \\
\text { goal setting; education; } \\
\text { motivational interviewing; } \\
\text { development of tailored health } \\
\text { education and individualized } \\
\text { plan and goals. Discuss healthy } \\
\text { weight measures and associated } \\
\text { risk factors i.e. BMl, waist/hip } \\
\text { ratio. Discuss menopause, } \\
\text { stress, sleep and other concerns. } \\
\text { Discuss appropriate screening. }\end{array}$ \\
\hline 3 (email) & & Email to check progress \\
\hline 6 & & $\begin{array}{l}\text { Review plan and goals; } \\
\text { Discuss personal action plan; } \\
\text { identify barriers, self-monitoring }\end{array}$ \\
\hline 12 & & $\begin{array}{l}\text { Reviews of plan and goals; } \\
\text { coaching; relapse prevention; } \\
\text { motivational interviewing; } \\
\text { biophysical measurements; } \\
\text { review observational weight } \\
\text { and self-measured height, } \\
\text { waist/hip circumference } \\
\text { measures. }\end{array}$ \\
\hline
\end{tabular}


about support services. No specific or individual advice was provided, as per usual practice.

\section{Quality assurance}

This study was conducted in accordance with the International Conference of Harmonisation Guideline E6 Good Clinical Practice (ICH-GCP) recommendations for clinical trials. Regular auditing of the adequacy and effectiveness of internal controls was undertaken0of risk management and compliance frameworks by the independent Clinical Trial Manager of the lead university.

To ensure the reliability of the anthropometrical measures and survey data collected by research assistants (RAs), standard operating procedures were developed. RAs also received a one-day training workshop prior to commencing data collection and were audited periodically by the research manager.

The fidelity of the nurse consultations was enhanced by ensuring that the trial nurses, who were experienced in cancer care, were also trained in the use of the intervention. Nurses received a self-directed protocol manual and DVD, and participated in a full day skills development session delivered by members of the research team. To ensure adherence to study protocols, peer review of virtual consultations was conducted by the clinical manager and nurses also completed a checklist at the end of each session to indicate the strategies used.

\section{Measurements}

Primary and secondary endpoints were measured at three time points (baseline, post-intervention or 12, and 24 weeks after the end of the trial) through a selfcompleted electronic survey or through RA data collection. Table 4 outlines the timing and mode of administration for each instrument. Data collectors were blinded to the group allocation of the participants.

\section{Primary endpoint}

The primary outcome measure was health-related quality of life as determined by the Functional Assessment of Cancer Therapy-General (FACT-G) [18], a 28-item instrument used extensively to assess satisfaction with the treatment relationship, physical, functional, social, and emotional well-being, and overall quality of life (QoL). Psychometric testing has shown good internal consistency (Cronbach alphas: physical sub-scale, 0.82; functional subscale, 0.80; social sub-scale, 0.69; emotional sub-scale, 0.72 ; and total score, 0.89$)$, test-retest reliability $(0.88,0.84$, $0.82,0.82,0.92$ respectively), and validity [18].

There is little consensus over the minimal clinically important difference (MCID) in health-related quality of life scores that constitutes a meaningful difference for participants (either beneficial or harmful), with MCID in the FACT-G total scores ranging from 4-8 points [19-
21]. Using a distribution-based approach, a 6 point or greater improvement in the FACT-G total scores (between $t_{0}$ and $t_{1}$ ) was considered a minimal clinically important change for women in the study [19-22].

\section{Secondary endpoints}

Changes in several secondary outcome measures were also assessed with the following instruments:

1. Menopausal symptoms were measured using the standard Greene Climacteric Scale@ [23], a 21-item scale that assesses self-reported vasomotor symptoms, somatic symptoms, psychological symptoms (anxiety and depression), and sexual function [23]. This scale has consistently demonstrated good psychometric properties and has been used in population-based and clinical samples in a variety of locales [23, 24].

2. Anthropometric measures were collected by RAs using standard protocols for the measurement of height and weight (from which BMI was derived), waist-to-hip ratio, and percentage of body fat. BMI was grouped according to the WHO International Classification of adult weight [25] (i.e., $<18.5$ being underweight, between 18.5 - 24.9 being in the normal weight range, and $\geq 30$ being obese).

3. Alcohol and tobacco use was assessed using several questions from the Australian Health Survey, a population-based survey designed that assesses the past and current patterns of consumption among the Australian population aged 18 and over [26].

4. Habitual dietary intake (including greater detail of alcohol consumption) was monitored through the Food Frequency Questionnaire (FFQ) [27, 28]. The FFQ covers dietary intake of cereal foods, sweets and snacks, dairy products, meats and fish, fruit, vegetables, and alcoholic beverages $[27,28]$ and is one of few dietary intake measures that minimises day-to-day variability by assessing usual dietary intake in the past 12-months.

5. Physical activity was measured using the validated International Physical Activity Questionnaire Short Form (IPAQ-SF) [29]. The short version of this instrument is recommended for use in national and regional surveillance systems [30]. It assesses three types of activity (walking, moderate-intensity activities and vigorous-intensity activities), which are all targeted behaviours within the intervention.

6. Sleep activity and quality were measured using the Pittsburgh Sleep Quality Index (PSQI), a 19-item self-report instrument with well-established reliability and validity [27]. The PSQI includes seven subscales (subjective sleep quality, sleep latency, sleep duration, habitual sleep efficiency, sleep disturbances, 
Table 4 Outcome measures, instruments, modes of administration, and time points of the study

\begin{tabular}{|c|c|c|c|c|c|c|}
\hline \multirow[t]{2}{*}{ Measures } & \multirow[t]{2}{*}{ Instruments } & \multirow[b]{2}{*}{$t_{0}$ (baseline) } & \multirow{2}{*}{$\begin{array}{l}\text { Time point } \\
t_{1} \text { (12 weeks) }\end{array}$} & \multirow[b]{2}{*}{$t_{2}$ (24 weeks) } & \multicolumn{2}{|c|}{ Mode of administration } \\
\hline & & & & & Online survey & RA data collection \\
\hline \multicolumn{7}{|l|}{ Background information } \\
\hline \multicolumn{7}{|l|}{ Socio-demographics } \\
\hline Medical and surgical history & & $x$ & \multirow{2}{*}{\multicolumn{2}{|c|}{$\begin{array}{l}\text { Assesses changes in medical/surgical history, } \\
\text { and medications since baseline }\end{array}$}} & & $x$ \\
\hline Cancer diagnosis and treatment & & $x$ & & & & $x$ \\
\hline Medications & & $x$ & & & & $x$ \\
\hline \multicolumn{7}{|l|}{ Subjective health indicators } \\
\hline \multirow[t]{2}{*}{ HRQoL } & FACT G [18] & $x$ & $x$ & $x$ & $x$ & \\
\hline & $S F-36[41]$ & $x$ & $x$ & $x$ & $x$ & \\
\hline Depression & CES - D [42] & & & & & \\
\hline Anxiety & Zung SAS [43] & & & & & \\
\hline \multicolumn{7}{|l|}{ Sexuality } \\
\hline Sexual function & FSFI [44] & $x$ & $x$ & $x$ & $x$ & \\
\hline Exercise self-efficacy & ESE [45] & $x$ & $x$ & $x$ & $x$ & \\
\hline Dietary self-efficacy & DSE [45] & $x$ & $x$ & $x$ & $x$ & \\
\hline \multicolumn{7}{|l|}{ Menopausal symptoms } \\
\hline Menopausal symptoms & GCS [23] & $x$ & $x$ & $x$ & $x$ & \\
\hline \multicolumn{7}{|l|}{ Modifiable lifestyle factors } \\
\hline Diet & FFQ $[46,47]$ & $x$ & $x$ & $x$ & & $x$ \\
\hline Physical Activity & IPAQ [48] & & & & & \\
\hline Sleep & PSQI [27] & & & & & \\
\hline Waist and hip circumference & & $x$ & $x$ & $x$ & & $x$ \\
\hline \multicolumn{7}{|l|}{ Cost effectiveness evaluation } \\
\hline Calendar of costs incurred & & $x$ & $x$ & $x$ & & $x$ \\
\hline
\end{tabular}

FACT G Functional Assessment of Cancer Therapy - General, SF - 36, Short Form 36, CES - D, Center for Epidemiologic Studies Depression Scale, Zung SAS Zung self-rating anxiety scale, FSFI Female Sexual Function Index, ESE Exercise Self-Efficacy, DSE Dietary Self-Efficacy, GCS Greene Climacteric Scale; FFQ Food Frequency Questionnaire, IPAQ International Physical Activity Questionnaire, PSQI Pittsburgh sleep quality index

use of sleeping medication, and daytime dysfunction) and an overall summary score and has demonstrated utility in both clinical and research settings [27].

\section{Data analysis}

\section{Sample size calculations}

A priori power analysis $[28,31]$ determined the minimum sample size required for each group is 268 , with a total sample size of 536 (calculation based on: standardised mean difference $(\mathrm{SMD})=0.65,90 \%$ power, $95 \%$ confidence interval; 25\% non-response). However, delays in ethical approval across sites and slower than anticipated recruitment meant the initial calculation were revised. Thus $A$ posteriori power analysis were performed and revealed that 250 participants (125 per group) were required to detect a standardised mean difference (SMD) of 0.6 or greater $[32,33]$ in the primary outcome measure (FACT-G total). Calculations were based on achieving $80 \%$ power, with a type I error of $5 \%$ (twotailed) to detect a 5.7 change in the standard deviation of the FACT-G total $(M=76.2, S D=14.3$ from pilot study [10]), and $25 \%$ non-adherence (10\% lost to followup and $15 \%$ non-response).

\section{Primary analysis}

All statistical data will be analysed using Statistical Package for the Social Sciences (SPSS) ${ }^{\circledR}$ version 23 [34] and STATA 13 [35] statistical packages in adherence with the CONSORT reporting guidelines [36]. Baseline measures and participant characteristics will be initially compared to assess for imbalances and differences between groups while the primary endpoint (HRQoL) will examine within- and between-group differences in FACT-G scores over the study period. Logistic regression models will examine predictors of quality of life (including baseline quality of life), age and sociodemographic variables. This includes exploring the rurality of the women according to the classifications of highly accessible, accessible, moderately accessible, remote, and very remote location suggested by the Accessibility/Remoteness Index of Australia (ARIA) [37]. Linear 
mixed models (LMM) will analyse between-group changes and changes over time in secondary outcomes such as energy, macronutrient and micronutrient intake, body fatness, menopausal symptoms, levels of physical activity, alcohol, and smoking.

\section{Discussion}

Recent research indicates that women diagnosed with blood, breast, and gynaecological cancers have a distinct set of health needs after treatment. For example, among breast cancer survivors menopausal symptoms, infertility, fatigue, lymphedema, and osteoporosis can persist after treatment and are likely to have a significant and negative affect on ongoing health and wellness [38]. In many instances, the health system does not adequately address these health issues and is struggling to support the increasing number of cancer survivors in the population who subsequently develop treatment-related chronic diseases [39].

This study seeks to trial the effectiveness of a digitised, multimodal lifestyle intervention for the management of treatment-induced late health effects in women after cancer. We used an e-health enabled platform to reduce accessibility issues associated with cost, time, geographical, and other constraints.

Close-out will occur November 2016, at which time data will be prepared for the longitudinal data analysis. The findings from this study will contribute to evidence-based information about the utility and benefits of structured health promotion activities for women after cancer.

\begin{abstract}
Abbreviations
ARIA: Accessibility/Remoteness index of Australia; BCNA: Breast cancer network Australia; BMI: Body mass index; FACT-G: Functional assessment of cancer therapy-general; FFQ: Food frequency questionnaire; HREC: Human research ethics committee; HRQOL: Health-related quality of life; HRT: Hormone replacement therapy; IPAQ: International physical activity questionnaire; KWC: Kim Walter's choices; LMM: Linear mixed models; NHMRC: National health and medical research council; QALYs: Quality of adjusted life years; QoL: Quality of life; RACGP: Royal Australian college of general practitioners; RAs: Research assistant's; RCT: Randomized controlled trial; SMD: Standardized mean difference; SPSS: Statistical package for the social sciences; WHO: World health organisation; WWACP: Women's wellness after cancer program; WWP: Women's wellness program
\end{abstract}

\section{Acknowledgements}

Acknowledgement and sincere thanks to the women who are enrolled in this study.

\section{Funding}

Funding for this research was received from the National Health and Medical Research Council (NHMRC) Partnership Projects Grant Application (APP1056856).

\section{Availability of data and materials}

Not applicable.

\section{Authors' contributions}

This study was designed by DA and ALM. The manuscript was written by CS, DA, ALM. DA is the coordinating investigator of the trial. DA and CS performed the sample size calculation. JPS is the clinical coordinator of the trial. AM and SB is the trial manager and made substantial contributions to the editing of the study protocol and the organization of this trial. DA, ALM,
$D T$, JT, LM, PY, NK, LY, KW, KL, SH, MK, KW are members of the scientific steering committee of the trial. DA, ALM, JPS, AM, SB, WS, CS are involved in trial implementation and critically revised the manuscript. All authors have read and approved the manuscript.

\section{Competing interests}

The authors declare that they have no competing interests.

\section{Consent for publication}

Not applicable.

\section{Ethics approval and consent to participate}

This study was approved by Queensland University of Technology Human Research Ethics Committee (Approval No: 1300000335) in July 2013. The study is also approved by the local ethics committees of all participating hospitals and health services (Peter MacCallum Cancer Centre, Metro South Hospital and Health Service, Royal Prince Alfred Hospital, St John of God Murdoch Hospital) and by the University of Notre Dame. The RCT has been registered with the Australian and New Zealand Clinical Trials Registry (Trial ID: ACTRN12614000800628). All participants provided written informed consent to participate in the trial.

\section{Author details}

${ }^{1}$ Menzies Health Institute Queensland, Griffith University, Menzies Health Institute Queensland, Parklands Drive, Southport, Queensland 4215, Australia. Institute of Health and Biomedical Innovation, Queensland University of Technology, Brisbane, Queensland, Australia. ${ }^{3}$ University of Queensland, Brisbane, Queensland, Australia. ${ }^{4}$ Royal Brisbane and Women's Hospital, Brisbane, Queensland, Australia. ${ }^{5}$ University of Notre Dame, Perth, Western Australia, Australia. ${ }^{6}$ St John of God Murdoch Hospital, Perth, Western Australia, Australia. ${ }^{7}$ Choices Cancer Support Program, Wesley Hospital, Brisbane, Queensland, Australia. ${ }^{8}$ Shanghai Ocean University, Shanghai, China. ${ }^{9}$ University of Sydney, Sydney, New South Wales, Australia. ${ }^{10}$ Royal Prince Alfred Hospital, Sydney, New South Wales, Australia. ${ }^{11}$ University of California San Francisco, California, USA. ${ }^{12}$ Central Queensland University, Brisbane, Queensland, Australia. ${ }^{13}$ Peter MacCallum Cancer Institute, Melbourne, Victoria, Australia. ${ }^{14}$ Breast Cancer Network Australia, Melbourne, Victoria, Australia. ${ }^{15}$ Division of Cancer Services, Princess Alexandra Hospital, Brisbane, Queensland, Australia.

Received: 1 July 2016 Accepted: 25 January 2017

Published online: 03 February 2017

\section{References}

1. Australian Institute of Health and Welfare (AlHW). Cancer in Australia 2014: an overview. Canberra: AlHW; 2015

2. Gupta P, Sturdee DW, Palin SL, Majumder K, Fear R, Marshall T, Paterson I. Menopausal symptoms in women treated for breast cancer: the prevalence and severity of symptoms and their perceived effects on quality of life. Climacteric. 2006:9(1):49-58.

3. Eakin EG, Youlden DR, Baade PD, Lawler SP, Reeves MM, Heyworth JS, Fritschi L. Health status of long-term cancer survivors: results from an Australian population-based sample. Cancer Epidemiol Biomarkers Prev. 2006;15(10):1969-76.

4. Evens K, Eschiti VS. Cognitive Effects of Cancer Treatment: "Chemo Brain" Explained. Clin J Oncol Nurs. 2009;13(6):661-66.

5. Bower JE, Ganz PA, Desmond KA, Bernaards C, Rowland JH, Meyerowitz BE, Belin TR. Fatigue in long-term breast carcinoma survivors: a longitudinal investigation. Cancer. 2006;106(4):751-8.

6. Blanchard CM, Courneya KS, Stein K. Cancer survivors' adherence to lifestyle behavior recommendations and associations with health-related quality of life: results from the American Cancer Society's SCS-II. J Clin Oncol. 2008; 26(13):2198-204.

7. Pollard A, Eakin E, Vardy J, Hawkes A. Health behaviour interventions for cancer survivors: An overview of the evidence and contemporary Australian trials. In: Cancer Forum: 2009. Sydney: The Cancer Council Australia; 2009. 184

8. Nathan PC, Ford JS, Henderson TO, Hudson MM, Emmons KM, Casillas JN, Lown EA, Ness KK, Oeffinger KC. Health behaviors, medical care, and interventions to promote healthy living in the childhood cancer survivor study cohort. J Clin Oncol. 2009;27(14):2363-73. 
9. McCarthy AL, Tramm R, Shaban RZ, Yates P. Factors influencing health behaviors of younger women after menopause-inducing cancer treatment. Public Health Nurs. 2013;30(2):106-16.

10. Anderson DJ, Seib C, McCarthy AL, Yates P, Porter-Steele J, McGuire A, Young L. Facilitating lifestyle changes to manage menopausal symptoms in women with breast cancer: a randomized controlled pilot trial of the pink Women's wellness program. Menopause. 2015;22(9):937-45.

11. Hewitt M, Greenfield S, Stovall E. From cancer patient to cancer survivor: lost in transition, Committee on cancer survivorship: improving care and quality of life, institute of medicine and national research council. Washington: The National Academies Press; 2006.

12. Anderson D, Seib C, McGuire A, Porter-Steele J. Decreasing menopausal symptoms in women undertaking a web-based multi-modal lifestyle intervention: the Women's wellness program. Maturitas. 2015;81(1):69-75.

13. Mirzaiinjmabadi $K$, Anderson D, Barnes M. The relationship between exercise, body mass index and menopausal symptoms in midlife Australian women. Int J Nurs Pract. 2006:12(1):28-34.

14. Karacan S. Effects of long-term aerobic exercise on physical fitness and postmenopausal symptoms with menopausal rating scale. Sci Sports. 2010; 25(1):39-46

15. Anderson DJ, Porter-Steele J, Yates PM, McCarthy AL, Whiteside E, Young $L$, Hargraves M, White KM, Byrne N, McCarthy N. Facilitating lifestyle changes to manage menopausal symptoms in women with breast cancer: delivering the pink Women's wellness program. Vienna: European Breast Cancer Conference; 2012

16. McLeod Al. Remark AS R58: a remark on algorithm AS 183. An efficient and portable pseudo-random number generator. J R Stat Soc: Ser C: Appl Stat. 1985;34(2):198-200.

17. Wichmann B, Hill D. Algorithm AS 183: an efficient and portable pseudorandom number generator. J R Stat Soc: Ser C: Appl Stat. 1982;31(2):188-90.

18. Cella DF, Tulsky DS, Gray G, Sarafian B, Linn E, Bonomi A, Silberman M, Yellen SB, Winicour P, Brannon J, et al. The functional assessment of cancer therapy scale: development and validation of the general measure. J Clin. 1993;11(3):570-9.

19. Cella D, Eton DT, Lai JS, Peterman AH, Merkel DE. Combining anchor and distribution-based methods to derive minimal clinically important differences on the functional assessment of cancer therapy (FACT) anemia and fatigue scales. J Pain Symptom Manag. 2002;24(6):547-61.

20. Cella D, Hahn EA, Dineen K. Meaningful change in cancer-specific quality of life scores: differences between improvement and worsening. Qual Life Res. 2002;11(3):207-21.

21. Eton DT, Cella D, Yost K, Yount SE, Peterman AH, Neuberg DS, Sledge GW, Wood WC. A combination of distribution- and anchor-based approaches determined minimally important differences (MIDs) for four endpoints in a breast cancer scale. J Clin Epidemiol. 2004;57(9):898-910.

22. Norman GR, Sloan JA, Wyrwich KW. Interpretation of changes in healthrelated quality of life: the remarkable universality of half a standard deviation. Med Care. 2003;41(5):582-92.

23. Greene JG. Constructing a standard climacteric scale. Maturitas. 1998;29(1):25-31.

24. Greene JG. Factor analyses of climacteric symptoms: toward a consensual measure. Glasgow: Department of Psychological Medicine, University of Glasgow; 1990.

25. World Health Organisation (WHO). Obesity: preventing and managing the global epidemic. Report of a WHO consultation. WHO technical report series 894. Geneva: WHO; 2000.

26. AlHW. 2010 national drug strategy household survey report. Drug statistics series no. 25. Canberra: AlHW; 2011

27. Buysse D, Reynolds C, Monk T, Berman S, Kupfer D. The Pittsburgh sleep quality index: a new instrument for psychiatric practice and research. Psychiatry Res. 1989;28:193-213.

28. Lehr R. Sixteen S-squared over D-squared: a relation for crude sample size estimates. Stat Med. 1992;11:1099-102.

29. Craig CL, Marshall AL, Sjostrom M, Bauman A, Booth ML, Ainsworth BE, Pratt M, Ekelund U, Yngve A, Sallis JF et al. International Physical Activity Questionnaire: 12-country reliability and validity. Med Sci in Sports Exerc. 2003;35(8):1381-95.

30. Booth M, Ainsworth B, Pratt M, Ekelund U, Yngve A, Sallis J, Oja P. International physical activity questionnaire: 12-country reliability and validity. Med Sci Sports Exerc. 2003;195(9131/03):3508-1381.

31. Norman G, Monteiro S, Salama S. Sample size calculations: should the emperor's clothes be off the peg or made to measure? BMJ 2012, 345
32. Brucker PS, Yost K, Cashy J, Webster K, Cella D. General population and cancer patient norms for the functional assessment of cancer therapygeneral (FACT-G). Eval Health Prof. 2005;28(2):192-211.

33. National Institute of Clinical Excellence (NICE). Depression: management of depression in primary and secondary care. Clinical practice guideline No 23. London: National Institute for Clinical Excellence; 2004.

34. IBM SPSS. IBM SPSS statistics for windows, version 22.0. Armonk: IBM Corp; 2013.

35. StataCorp. Stata statistical software: release 13. In: College station. TX: StataCorp LP; 2013.

36. Schulz KF, Altman DG, Moher D. CONSORT 2010 Statement: updated guidelines for reporting parallel group randomised trials. BMC Med. 2010; $8(1): 1-9$.

37. (AlHW) AloHaW. Rural, regional and remote health: a guide to remoteness. Canberra: AlHW; 2011.

38. Ganz P, Hahn E. Implementing a survivorship care plan for patients with breast cancer. J Clin Oncol. 2008;26(5):759-67.

39. Department of Health. Living with \& beyond cancer: taking action to improve outcomes (an update to the 2010 the national cancer survivorship initiative vision). London: Department of Health; 2013.

40. World Cancer Research Fund/American Institute for Cancer Research. Food, nutrition, physical activity, and the prevention of cancer: a global perspective. Washington: AICR; 2007.

41. Ware J, Sherbourne C. The MOS 36-Item Short-Form Health Survey (SF-36 ${ }^{\circledR}$ ): I. conceptual framework and item selection. Med Care. 1992;30(6):473-83.

42. Radloff LS. The CES-D scale: a self-report depression scale for research in the general population. Appl Psychol Meas. 1977;1(3):385-401.

43. Zung WW. A rating instrument for anxiety disorders. Psychosomatics. 1971; 12(6):371-9.

44. Rosen R, Brown C, Heiman J, Leiblum S, Meston C, Shabsigh R, Ferguson D, D'Agostino RJ. The female sexual function index (FSFI): a multi-dimensional self-report instrument for the assessment of female sexual function. J Sex Marital Ther. 2000;26(2):191-208.

45. Bandura A. The primacy of self-regulation in health promotion. Appl Psychol. 2005;54(2):245-54

46. Cancer Council Victoria. In: Centre CE, editor. Dietary questionnaire for epidemiological studies (DQES v2): user information guide. Carlton: Cancer Council Victoria; 2009.

47. Giles GG, Ireland PD, Giles GG, Ireland PD. Dietary questionnaire for epidemiological studies (version 2). Melbourne: The Cancer Council Victoria; 1996

48. Craig CL, Marshall AL, SjÖstrÖm M, Bauman AE, Booth ML, Ainsworth BE, Pratt M, Ekelund U, Yngve A, Sallis JF, et al. International physical activity questionnaire: 12-country reliability and validity. Med Sci Sports Exerc. 2003; 35(8):1381-95.

\section{Submit your next manuscript to BioMed Central and we will help you at every step:}

- We accept pre-submission inquiries

- Our selector tool helps you to find the most relevant journal

- We provide round the clock customer support

- Convenient online submission

- Thorough peer review

- Inclusion in PubMed and all major indexing services

- Maximum visibility for your research

Submit your manuscript at www.biomedcentral.com/submit 